\title{
VARIATIONS OF INFERIOR ALVEOLAR NERVE AND ITS RELATION WITH MAXILLARY ARTERY IN INFRA TEMPORAL FOSSA
}

\begin{tabular}{|c|}
\hline Anatomy \\
\hline
\end{tabular}

Dr. Ambili. P

Associate Professor (CAP), Department Of Anatomy, Govt. Medical College Kozhikode, Kozhikode, Kerala, India.

\section{ABSTRACT}

Infra temporal fossa (ITF) is a difficult area to access both in anatomical dissection and surgery. Thorough knowledge on the anatomy of infra temporal fossa is a prerequisite for maxillofacial, ENT and neurosurgeons since it is in close relation with oral \& nasal cavities, maxillary antrum, orbit \& pharynx and has direct access to skull base \& cranial cavity. Moreover it is a difficult area to describe because of lack of well defined boundary. It contains mandibular nerve (MN), maxillary artery (MA), otic ganglion, chorda tympani nerve and venous plexus apart from pterygoid muscles for mastication.

Some variations of the structures in the fossa were noted during routine dissection. This study focuses on such variations of the Inferior Alveolar Nerve and its relation with the maxillary artery in the infra temporal fossa. Present study was done in 60 ITF from 30 cadavers. Variations were observed in 9 specimens - 2 bilaterally, 3 on right side and 2 on left side - in connection with their mode of origin and communication with other nerves. Variations were also observed in their length, thickness and relation with MA. A peculiar branching pattern of MA has been described. Possible embryological basis of such variations has been discussed.

\section{KEYWORDS}

Inferior Alveolar Nerve, Infra temporal Fossa, Maxillary Artery, Variations

\section{INTRODUCTION:}

ITF is covered laterally by the ramus of mandible, anteriorly by the maxilla and superiorly by the sphenoid. It communicates with Orbit, middle cranial fossa and nasopharynx. Mandibular nerve (MN), Maxillary artery (MA), otic ganglion and chorda tympani nerve are the contents apart from the pterygoid muscles. This fossa is often approached by neurosurgeons for accessing the skull base, oral and maxillofacial surgeons and reconstructive surgeons for administering nerve blocks, by onco surgeons for management of primary and secondary tumors and radiologists for maxillary artery embolization. The variations in the anatomy of structures in the fossa should be familiarised to avoid unnecessary complications and to anticipate possible complications.

$\mathrm{MN}$ is the only mixed branch of Trigeminal Nerve. Variations of branches of MN have been reported by investigators regarding their mode of origin, communication with other cranial nerves and with branches of the same nerve. Variation in the course and branching pattern of MA and its relation with the branches of MN also has been extensively studied. IAN has been studied in detail may be because it is frequently encountered in dental interventions. Inadequate anaesthesia during IAN block, maxillary blanching and pale cheek, and numbness of external ear has been reported after IAN block. Orofacial pain, tic douloureux and some type of migraine have been attributed to the variations in the vascular relation of the nerve by the investigators.

\section{METHODS:}

The study was done in 60 ITF from 30 cadavers ( 16 males and 4 females) over a period of 2 years in Govt Medical College, Kozhikode ITF has been exposed by removing the ramus of mandible. Maxillary A and its relation with lateral pterygoid, thickness of IAN and Lingual $\mathrm{N}$ were observed followed by the removal of lateral pterygoid muscle. Main trunk of $\mathrm{MN}$ emerging from foramen ovale was identified. Its length and branching patterns were noted. Mode of origin, length and thickness of IAN, its communication with other nerves and its relation with MA were studied in detail.

The patterns identified were represented by diagrams and the photographs of the dissected specimens were taken. The data were converted into charts and the frequency was calculated. It was compared with observations from previous studies.

\section{RESULTS:}

\section{Origin of IAN from MN}

Present study showed that in majority of the specimens dissected $(88 \%)$, the posterior division of MN bifurcated into IAN and Lingual $\mathrm{N}$. The origin of IAN as a bifurcation was at a distance varying between $6 \mathrm{~mm}$ and $22 \mathrm{~mm}$ distal to the foramen ovale. In the specimens with IAN having more than one root, the proximal root was still closer to the foramen $(3 \mathrm{~mm})$.

\section{Mode of origin of IAN}

In majority of the specimens (53/60) IAN took origin as bifurcation from MN, along with Lingual N. 6/60 showed origin of Inferior alveolar nerve by 2 roots (Fig 1,3,4). Source of origin of second root varied. It was observed to arise from ATN, either from its inferior root $(2 / 6)$ or as a common twig bifurcating $(2 / 6)$ or directly from the MN $(2 / 6)$

Fig1: IAN with 2 roots - one as bifurcation from MN, one as a thin common twig arising from $\mathrm{MN}$ which bifurcated and joined with IAN and ATN. ATN has 3 roots and MMA passed lateral to all the 3 roots
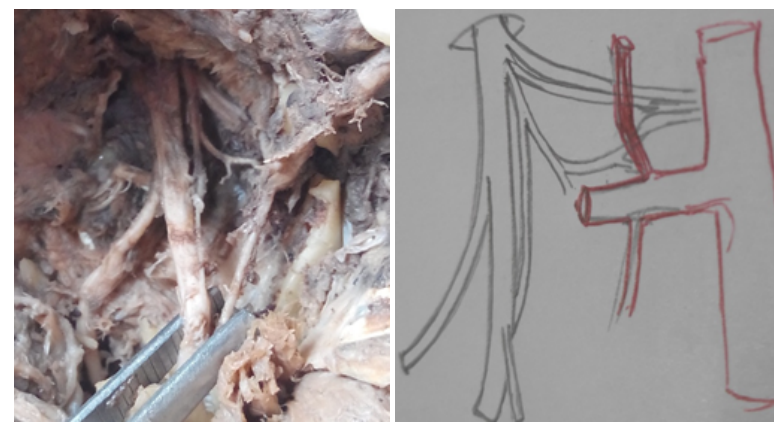

$1 / 60$ showed 3 roots for IAN (Fig 2). First root as bifurcation from MN, second root was from the $\mathrm{MN}$ close to the origin of ATN, which in turn had only one root. Third root was from ATN distal to MMA. Here the third root can be considered as the inferior root of ATN arising from IAN.



Fig 2: IAN with 3 roots - one as bifurcation from MN, one from MN close to origin of ATN, one from ATN distal to MMA

\section{Relation with Maxillary A}

MA was superficial to the Lat pterygoid in 42 specimens (70\%). In 12 specimens, the MA was deep to Lat pterygoid and lateral to IAN (20\%). In 5 specimens (8.3\%), it was deep to Lat Pterygoid but passed through the nerve $(2 / 60)$ or the roots of the nerve. In one showed a peculiar arrangement. 
2/60 specimens, IAN was pierced by MA $2 \mathrm{~mm}$ inferior to its origin from the MN. In one specimen, the thick superficial part was anterior and in the other it was posterior, when compared to the part deep to the MA.

3/6 specimens with 2 roots for IAN, MA passed through the loop formed by the roots of IAN. In one specimen, MA passed between 2 roots of ATN in addition to IAN (Fig 3). 2/6 MA passed lateral to the 2 roots.

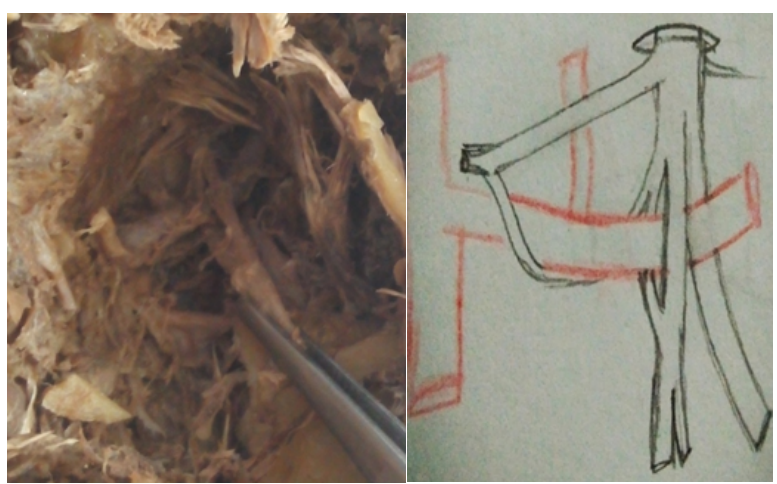

Fig 3: IAN with 2 roots - deep root in common with inferior root of ATN and superficial root as bifurcation from MN. MA passes through the 2 roots of ATN and 2 roots of IAN. MMA is deep to superior root of ATN A peculiar arrangement of Maxillary artery was observed in one Right ITF (1/6) where MA was divided into superficial and deep branches. This deep branch passed through the loop formed by ATN above, IAN anteriorly and the communication between the two posteriorly (Fig 4) and after giving the MMA it left the fossa through foramen ovale.

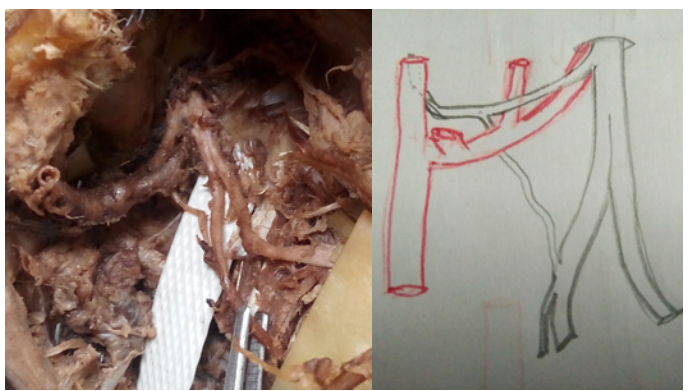

Fig 4: IAN with 2 roots - one as bifurcation from $\mathrm{MN}$ and one from ATN. ATN was single rooted. MA divided into superficial and deep. The deep branch passed through the loop and entered F.Ovale. MMA from this deep branch passed behind the ATN.

In $1 / 60$ specimens, IAN with 3 roots, MMA was passing through the loop formed by ATN, IAN and the root from ATN (Fig 2).

\section{DISCUSSION:}

IAN is otherwise known as the dentist's nerve. IAN block is used for many surgical procedures on mandible, the commonest being the molar tooth extraction. The origin of IAN in the present study was as described in the text books in $88 \%$ as one of the two terminal branches from the posterior division of MN.

Kim et al (2004) reported the average distance of bifurcation as $14.3 \mathrm{~mm}$ from foramen ovale and Jeerapat Singam et al (2015) as $11.6 \pm$ $4.12 \mathrm{~mm}$. In the present study it was 6 to $22 \mathrm{~mm}$ distal to the foramen ovale.

Regarding the mode of origin of IAN, origin by 2 roots was the most frequently reported variation (Roy et al 2002, Anil et al 2003, Zaidi \& Hanif 2010, Sharma et al2011). It was seen 6/60 in the present study.

The IAN with 3 roots similar to one in the present study, taking origin from the bifurcation, trunk of MN and ATN, was reported by MM Pai et al (2010), Thotakura et al (2013) and Sumalatha et al (2018). The ATN was originating as a single root in all these cases as well. Quadros (2013) described the IAN with 3roots, 2 roots from the lower root of ATN and 1 from Lingual.
Communication of IAN with ATN was reported in $4 / 32$ by Kim et al (2004) and $5 / 70$ by Jeerapat Singam et al (2015). It was $5 / 60$ in the present study, either as origin from the nerve or a twig bifurcated to join the ATN and IAN.

These communications between the branches of MN can be the alternative route for secretomotor fibres as suggested by Thotakura et al (2013). The same can be considered to explain the pain felt along the distribution of any of these branches without a reason as suggested by Siessere et al (2009).

This may explain the temporary numbness of external ear and the region supplied by ATN as reported after IAN block by Ngeow \& Chai (2009). It can explain the incomplete nerve block in dental anaesthesia or inadvertent effect on infiltrating the local anaesthetic drug.

The variations observed can be explained by the theories of neural crest cell migration during embryogenesis as suggested by Bronner Fraser (1993). Neural crest cells migrate from the cephalic region through mesoderm of first pharyngeal arch with the help of multiple cell matrix interactions, contact repulsion and chemo repulsion to give rise to the MN in ITF. Several inhibitory factors released from the caudal somites plays a role in its migration. In the embryo the MN passes through the vascular network of MA.

$\mathrm{MN}$ is the only branch from the trigeminal nerve that contain both motor and sensory fibres and it is possible that these fibres may take separate paths during migration and may explain the variation in the formation and communication between the branches of MN

Topographical relation of MA with the structures in ITF is clinically significant. The MA here can be ligated for controlling posterior epistaxis trans orally as reported by Dennis R Maceri (1984). This artery can be used for embolization prior to onco surgical procedures. Micro vascular decompression has been reported as surgical treatment for trigeminal neuralgia/ tic douloureux and migraine (Siéssere 2009).

According to Compendium of human anatomic variation by Bergman et al (1988) Second part of MA was superficial in $69.5 \%$. Second part of MA was reported to have found superficial to the lower head of lateral pterygoid muscle by Hussain et al (2008) in $71 \%$ men, Standring et al (2008) in 60\% and Uysal et al (2011) in 57.2\%. Present study showed a similar result where MA was superficial in $71 \%$ of the total specimens. It was similar to the frequency of Caucasoids as cited by Maeda et al (2012)

MA piercing the IAN was reported in 1/80 ITF by Roy et al (2002), in $3 / 50$ by Khan et al (2010) and one case by Zaidi \& Hanif (2010). In the present study MA pierced the IAN in 2/60 and MA passed between the 2 roots of IAN, one root from ATN in 2/60. Similar finding of MA passing between the 2 roots were reported by Anil et al (2003) in 2/20, Bharadwaj et al (2014) in 2/30, Sobhakumari et al B/L (2015).

This peculiar relation of the artery is clinically important as the undue traction of the IAN during or after a surgical procedure can lead to arterial compression and vascular compromise distal to that site. It also can lead to damage of deep root of IAN during arterial ligation. The pulsations of the artery causing abnormal sensation along the nerve also should be considered.

Maeda et al (2012) in a study in 208 Japanese people, reported a case where MA divided into superficial and deep. The MMA \& AMMA originated from it and reunited with superficial. The author refers similar findings by Lauber (1901) and Claire et al (2011). A similar case was reported by Tadokaoro et al (2008) but the deep branch was larger and it continued into pterygopalatine fossa. In the present study, MA was divided into superficial and deep branches almost of the same calibre (Fig 4). The deep branch gave rise to MMA and entered the F Ovale. Inferior alveolar artery was a branch from superficial part in all these cases as in the present study.

As cited by Roy et al, the hemodynamic balance between the developing mandibular and meningeal blood vessels play an important role in shaping the MA course in the ITF (Biermann 1943, Hogg et al 1972). The initial arterial supply of this region was by the stapedial artery, which is carried distally with the developing MN and feeds the vascular network formed in the pterygoid myoblasts. Later it was fed by ECA. It was followed by the disappearance and persistence of several parts of these arterial loops establishing the adult pattern. 
This complex mechanism of formation of MA along with migrating neural crest cells in separate paths may be a possible explanation for the different arterial patterns and relations with the nerves observed in the study.

\section{CONCLUSION:}

Variations were observed in the structures in ITF, in regard to the mode of origin of IAN and its relation with the maxillary artery. The awareness of possible variations in the structures in ITF is important especially in Neuro, ENT and Onco and Oro-maxillofacial surgeons. Surgical approach in the ITF needs a thorough knowledge of the anatomy of this region in order to prevent unnecessary arterial or neurological complications. These types of variations give an insight into the embryological origin of the neural tissue and the vascular pattern observed here.

\section{REFERENCES:}

1. Anil A, Peker T et al. Variations in the anatomy of Inferior Alveolar Nerve. Br J Oral Maxillofac Surg (2003) 41(4): 236-239

2. Balaji Thotakura, Rajendran SS et al. Variation in posterior division of Mandibular Nerve in cadavers. Singapore Med J (2013) 54(3): 149-151

3. Bronner - Fraser M. 1993 . Environmental influences on Neural crest cell migration J Neurobiol. 24. 233-247.

4. Cosmin Ioan Mohor. The Maxillary Artery - Importance of topographic relations. Acta Medica Transilvanica (2014) 2(3): 235-237

5. Jeerapat Singsorn. A Morphological Study of Posterior Divisional Branches of the Mandibular Nerve in Embalmed Thai Cadavers 30(4). 2015.358 - 363

6. Khan MM et al. perforation of IAN with Maxillary Artery. Br J Oral Maxillofac Surg (2010) 48(8): 645-647

7. Lydia S Qudros et al. IAN with multiple roots. British Biomedical Bulletin (2014) 2(1): Lydias

8. Maeda, S., Aizawa, Y., Kumaki, K. et al. Variations in the course of the maxillary artery in Japanese adults. Anat Sci Int 87, 187-194 (2012).

9. Mangala M Pai, Swamy RS, Prabhu LV. Variation in morphology of IAN \& its clinical significance. Biomedicine International (2010) 1:93-95.

10. Nikha Bharadwaj, Sahni P et al. Anomalous communication between IAN \& ATN. Malays J Med Sci(2014) 21(5): 71-74

11. S Sumalatha, S R Kotian, H Thodety et al. Variations of the lingual and inferior alveolar nerves and their anomalous relationship with the maxillary artery. Eur. J. Anat. 22 (5): nerves and their

12. Sharma et al. unusual origin \& relation of IAN in left infra temporal region. J Indian Med Assoc (2011) 109(2): 96-98

13. Shobhakumari et al. Bilateral communication btwn branches of posterior division of Mand N \& clinical significance. J Dent and Med Sci (2015) 14(8): 36-39

14. Siéssere S, Hallak Regalo SC, Semprini M, et al. Anatomical variations of the mandibular nerve and its branches correlated to clinical situations. Minerva Stomatol 2009; 58:20 9-15

15. Tadokoro O, Umemura $\mathrm{Y}$, Utsuno $\mathrm{H}$, Inoue $\mathrm{K}$ (2008) A case of a divided maxillary artery in the infratemporal fossa. Okajimas Folia Anat Jan 85:97-101

16. TS Roy, AK Sarkar \& HK Panicker . Variation in the origin of IAN. Clinical Anatomy (2002) 15:143-147

17. WC Ngeow \& WL Chai. Numbness of ear following IAN block:the forgotten complication. Br Dent J (2009) 207: 19-21 